\title{
Mechanical behaviour and formation process of silkworm silk gut
}

\author{
José L. Cenis, Rodrigo Madurga, Salvador D. Aznar-Cervantes, \\ A. Abel Lozano-Pérez, Núria Marí-Buyé, Luis Meseguer-Olmo, \\ Gustavo R. Plaza, Gustavo V. Guinea, Manuel Elices, Francisco Del Pozo and \\ José Pérez-Rigueiro \\ High performance silk fibers were produced directly from the silk glands of silkworms (Bombyx mori) \\ following an alternative route to natural spinning. This route is based on a traditional procedure that \\ consists of soaking the silk glands in a vinegar solution and stretching them by hand leading to the \\ so called silkworm guts. Here we present, to the authors' best knowledge, the first comprehensive study \\ on the formation, properties and microstructure of silkworm gut fibers. Comparison of the tensile \\ properties and microstructural organization of the silkworm guts with those of naturally spun fibers \\ allows gain of a deeper insight into the mechanisms that lead to the formation of the fiber, as well as \\ the relationship between the microstructure and properties of these materials. In this regard, it is proved \\ that an acidic environment and subsequent application of tensile stress in the range of $1000 \mathrm{kPa}$ are \\ sufficient conditions for the formation of a silk fiber.
}

\section{Introduction}

Silks produced by arthropods are listed among the most outstanding materials in terms of their processing and performance. ${ }^{1-3}$ However, the extreme optimization of the natural system and its peculiarities has represented a major challenge to all attempts intended to disentangle the individual effects of its constituents in the spinning of silk. ${ }^{4}$ In particular, the roles of the chemical structure of the proteins, and of the physiological and anatomical features related to fiber processing have proven difficult to unravel, severely hampering a possible biomimetic ${ }^{5}$ approach based on these materials. .,7 $^{\text {a }}$

Any opportunity to uncouple the different elements that take part in the spinning process would allow clarification of many of the underlying principles of these materials. In this regard, the production of fibers directly from the silkworm silk glands, an alternative procedure to natural spinning known for several centuries, is a promising re-discovered field. This fiber is denominated silkworm (silk) gut and was widely used in angling as a leader in fly fishing and, to a lesser extent, as surgical sutures. Silkworm gut is obtained directly by the chemical and mechanical processing of the sericigen glands of the Bombyx mori silkworm. Traditionally, the method consisted of a previous immersion of 5 th instar larvae, just before spinning the cocoon, in a vinegar solution for several hours. Afterwards, glands were extracted manually from the worm's body and stretched from the two ends until resistance was found, indicating that the fiber was formed. The resulting fiber has a diameter of between 0.20 and $0.55 \mathrm{~mm}$, a length between 50 and $60 \mathrm{~cm}$ and a maximum force at breaking of $5 \mathrm{kgf}$.

Silkworm guts were highly appreciated due to their considerable tensile strength, and performed functions similar to modern polymeric fibers, especially in the fields of sutures and angling. A flourishing industry established around silkworm gut, which was located in the Spanish town of Murcia. Silkworm gut was completely displaced by nylon and other polymeric fibers around the 1940s, and its production was completely forgotten. However, there is interesting literature about this fiber in the field of angling. Apart from the classical photographic report by L. Marden, ${ }^{8}$ other authors have described the historical aspects of the fiber. ${ }^{9-11}$

The origins of silkworm gut are unclear. According to the cited sources, the first mention of the use of the term in angling dates back to 1722 in England. At the time, the origin of the production seemed to be Switzerland and north Italy. But progressively the Spanish town of Murcia, capital of the province of the same name in the south-east of Spain, became the world's 
only center for the manufacture of silk gut leaders. At peak production, Murcia produced 90 million strands of gut each year. According to Humphries, ${ }^{9}$ all major fishing-tackle producers had their agencies set up in Murcia, and most of the local production was exported to England. Silkworm gut was an inherently variable product, since its characteristics may vary with race, feeding and size of the silkworm. Consequently, the raw silkworm gut had to undergo a long process of cleaning of cellular debris and sericin, manual sorting and drawing through a perforated steel plate, in order to produce threads of consistent diameter and resistance. At least 17 different qualities of gut were produced, with diameters as small as $0.11 \mathrm{~mm}$. In a sense, its early application as a suture might be considered as an anticipation of the present boom of medical applications based on silkworm silk and related materials. $^{12-14}$

Paradoxically, the first systematic analysis of the silk gut formation process and the properties of the fibers was not performed on silkworms, but extrapolated to spider silk ${ }^{15}$ in an attempt to (a) check if the whole process could be applied to the major ampullate (MA) gland of Orbiculariae spiders, (b) gain insight in the spinning mechanism of MA silk by spiders. The success of this approach has led to the present work with two aims: first, to recover the traditional fabrication of silkworm gut to add this material to the present portfolio of silk biomaterials; and second, to use the formation process of silkworm gut in combination with its thorough mechanical and microstructural characterization in order to gain a deeper insight into both the processing and microstructure-property relationships of silkworm silk.

\section{Experimental section}

A batch of silkworms of the race Murcian White was reared on a natural diet of mulberry leaves in the facilities of IMIDA (Murcia, Spain). Just before starting the spinning process, the larvae were anaesthetized by exposure to a temperature of $4{ }^{\circ} \mathrm{C}$ for 15 minutes. The head of the worm was excised with a scalpel and the internal pressure of the body expulsed cleanly the two sericigen glands. The glands were washed and stored in distilled water until being processed.

The sericigen gland has a mucus-like texture and breaks up readily if stretched while being held with a couple of tweezers. However, the behaviour of the gland changes completely when incubated in an acidic environment, and eventually a resistant solid fiber is obtained upon subsequent stretching. The glands were immersed in acetic acid solutions of varying concentrations ( 0.5 and $8 \%$ acetic acid/water (v/v)) and for different times ranging from 0.5 to 20 minutes.

After removal from the acetic acid solution, the glands were stretched in a tensile testing machine (Instron 4411). A balance AND 1200 (resolution $\pm 10 \mathrm{mg}$ ) was used to measure the force exerted on the gland during stretching. A couple of tweezers were used as upper and lower grips for silk gut formation. The length of the gland between both tweezers was fixed to $60 \mathrm{~mm}$. Stretching proceeded at a constant speed of $500 \mathrm{~mm} \mathrm{~min}^{-1}$ until the silkworm gut detached from one of the tweezers. The process was characterized by the strain during formation, $e_{\mathrm{f}}$, defined as:

$$
e_{\mathrm{f}}=\frac{\Delta L}{L_{0}}
$$

where $L_{0}$ is the initial length of the gland between the tweezers (typically $60 \mathrm{~mm}$ ) and $\Delta L$ is the increment in length during the process, which was considered equal to the displacement of the crosshead. Values of the formation strain in the range $e_{\mathrm{f}}=5-10$ were found. In order to analyze the formation process at intermediate deformation stages, some samples were stretched up to a value of $e_{\mathrm{f}}=1$ (i.e. doubling their initial length) and are referred to as pre-gut fibers. In these cases, the samples did not detach from the grips.

$50 \mathrm{~mm}$ samples were cut from silkworm guts. The apparent diameter of each sample was measured with an optical microscope (Leica DMI 3000B) and the cross sectional area was calculated from the apparent diameter assuming a circular geometry. Silk gut samples were tested in air in an Instron 5866 tensile testing machine. Loads were measured with a $100 \mathrm{~N}$ load cell and the deformation of the fiber was considered equal to the displacement of the crosshead. Samples were tested at a speed of $1 \mathrm{~mm} \mathrm{~min}^{-1}$ under the nominal conditions of $T=23{ }^{\circ} \mathrm{C}$ and $\mathrm{RH}=40 \%$. Force-displacement curves were converted into engineering stress-engineering strain curves using the calculated cross sectional areas, and the initial fiber length.

The microstructures of silkworm silk guts processed under different conditions were studied by Attenuated Total ReflectionFourier Transform Infrared Spectroscopy (ATR-FTIR) and X-ray diffraction (XRD). ATR-FTIR spectra were obtained in the range of 550-4000 $\mathrm{cm}^{-1}$ in a Nicolet iS5 FT-IR spectrometer with an ATR module under observation conditions: 64 scans per spectrum with a resolution of $4 \mathrm{~cm}^{-1}$.

$\mathrm{XRD}$ was performed at room temperature in a Bruker Smart 1000 CCD diffractometer using graphite-monochromated Mo-K $\alpha$ radiation $(\lambda=0.71073 \AA)$ operating at $50 \mathrm{kV}$ and $30 \mathrm{~mA}$. Each still exposure was taken with a variable time ranging from 100 to 800 seconds depending on the diameter of the fiber. Sample to detector distance was calibrated with $\mathrm{Si}$ powder (NIST SRM640d). Patterns with no sample were used as background and subtracted from the patterns of the fibers. Two images were averaged for each sample. A region containing the main equatorial reflections (020) and (210) was azimuthally integrated resulting in a 1D profile. This profile was fitted with Gaussian functions for Bragg peaks and a short-range order halo, and a constant value for the residual background scattering of the sample. ${ }^{16}$

The position of the Gaussian functions which correspond to the (020), (210) and (002) reflections were used to calculate the unit cell parameters $a, b$ and $c$ of the $\beta$-sheet nanocrystals, which correspond to the interchain (hydrogen bonding), to the intersheet (piling-up of $\beta$-sheets) and to the protein backbone directions, respectively. The calculation assumed an orthorhombic geometry of the unit cell ${ }^{17}$ and was based on Bragg's equation $n \lambda=2 d \sin \theta$. 
Two different indexes were used to quantify the crystallinity of the fibers. The calculation of the $X$ crystalline index ${ }^{16}$ requires the azimuthal integration of the XRD pattern and the fitting of the integrated intensity profile with four sharp Gaussian functions, one broad Gaussian function and a constant. $X$ is defined as the ratio, $X=\sum I_{\mathrm{Bragg}} / I_{\mathrm{T}}$, where $\sum I_{\mathrm{Bragg}}$ is the sum of the intensities of the five Gaussians and $I_{\mathrm{T}}$ the total integrated intensity. Crystallinity was also calculated as $\chi$ from the intensity of the whole diffraction pattern, $I_{\mathrm{T}}(Q)$ as a function of the magnitude of the scattering vector $Q=4 \pi \sin (\theta) / \lambda$. In this case, the azimuthally averaged intensity of the amorphous halo, $I_{\mathrm{A}}(Q)$, was calculated by azimuthal integration of selected regions where the intensity arising from the Bragg peaks can be assumed to be negligible. The intensity of the Bragg peaks, $i_{\mathrm{c}}(Q)$, was then obtained as: $i_{\mathrm{c}}(Q)=I_{\mathrm{T}}(Q)-I_{\mathrm{A}}(Q) .{ }^{18}$ The ratio between the integrated intensity of the Bragg peaks and the integrated total intensity defines the $\chi$ parameter as:

$$
\chi=\frac{\int i_{\mathrm{c}}(Q) \mathrm{d} Q}{\int I_{\mathrm{T}}(Q) \mathrm{d} Q}
$$

The nanocrystal size along each crystallographic direction was obtained from Scherrer's equation, $L=(0.9 \lambda) /(B \cos \theta),{ }^{19}$ where $B$ is the full width at half maximum (FWHM) in the radial direction of the Gaussian fitted to the diffraction spot.

The orientation of the nanocrystals was measured as the full width at half maximum (FWHM) of the Gaussian used to fit the equatorial peaks (210) in the azimuthal direction. ${ }^{20}$

\section{Results and discussion}

\section{Geometry and dimensions of silkworm gut}

The silkworm guts show a smooth lateral surface as illustrated in Fig. 1a with diameters in the range of 400-600 $\mu \mathrm{m}$. Variations as large as $\pm 20 \%$ from the mean diameter can be found along the fiber (i.e. average diameter $D=480 \mu \mathrm{m}, D_{\max }=580 \mu \mathrm{m}$, $D_{\min }=370 \mu \mathrm{m}$, all values measured along a single silkworm gut). The cross sectional area of the silkworm gut is approximately circular, as illustrated in Fig. 1b. The size and cross sectional area of silkworm gut contrasts with those of naturally spun silkworm silk fibers, which are characterized by diameters in the range of $D \sim 8 \mu \mathrm{m}$ and a cross sectional area that can be approximated to an ellipse. ${ }^{21}$

\section{Tensile behaviour of spider silk gut}

The tensile properties of silkworm silk guts are presented in Fig. 2, classified by the duration of immersion in the acetic acid solution (Fig. 2a) or by the concentration of acetic acid in the solution (Fig. 2b). Silkworm guts prepared after immersion in a $2 \%$ acetic acid solution for 2 minutes were taken as a reference in both cases. Silkworm guts can be considered as high performance fibers according to the conventional condition of work to fracture, $W_{\mathrm{f}} \geq 50 \mathrm{MJ} \mathrm{m}{ }^{-3}$ (which corresponds to the work to fracture of Kevlar fibers ${ }^{22}$ ). In particular, values as high as $90 \mathrm{MJ} \mathrm{m}^{-3}$ are found in some of the tested fibers. Silkworm gut stress-strain curves seem to be relatively independent of the acetic acid concentration and immersion time except for the longest treatment $(2 \%, 10 \mathrm{~min})$ and highest concentration (4\%, $2 \mathrm{~min}$ ) conditions. In both cases, the stress-strain curves present a clear relative maximum at the end of the elastic regime $\left(\varepsilon_{\mathrm{y}} \sim 0.03\right)$ and the stress reached at a given value of strain is consistently lower than those observed in any other silkworm guts. It is also remarkable that the maximum strain at breaking measured from any of the silk guts $\left(\varepsilon_{\mathrm{u}}=0.58\right)$ corresponds to one of these $(4 \%, 2 \mathrm{~min})$ samples.

High performance is a characteristic of silkworm silk fibers ${ }^{23}$ although the comparison of naturally spun silk and silk guts shows some remarkable differences as illustrated in Fig. 2c. Comparison of the tensile properties of representative silkworm silk guts and those of silkworm silk fibers obtained either by forced silking ${ }^{24,25}$ or after a degumming treatment ${ }^{26}$ shows that the former can present higher values of strain at breaking but at the expense of lower values of tensile strength. From these results a first significant difference can be established when silkworm silk gut and spider silk gut are compared with their natural counterparts. It was found ${ }^{15}$ that spider silk gut corresponds to spider silk fiber, except for the larger cross sectional area of the former, while silkworm silk gut does not correspond exactly to the native material spun by the worm, despite sharing a common composition. The main mechanical parameters of silkworm silk guts and naturally spun silkworm silk fibers are summarized in Table 1.

Finally, Fig. 2d is included to illustrate the huge differences in the tensile properties between the mechanical behaviour of natural silkworm and silkworm guts when expressed in terms of force instead of stress due to the large differences in the diameters of both types of fibers. The 10000 -fold difference in
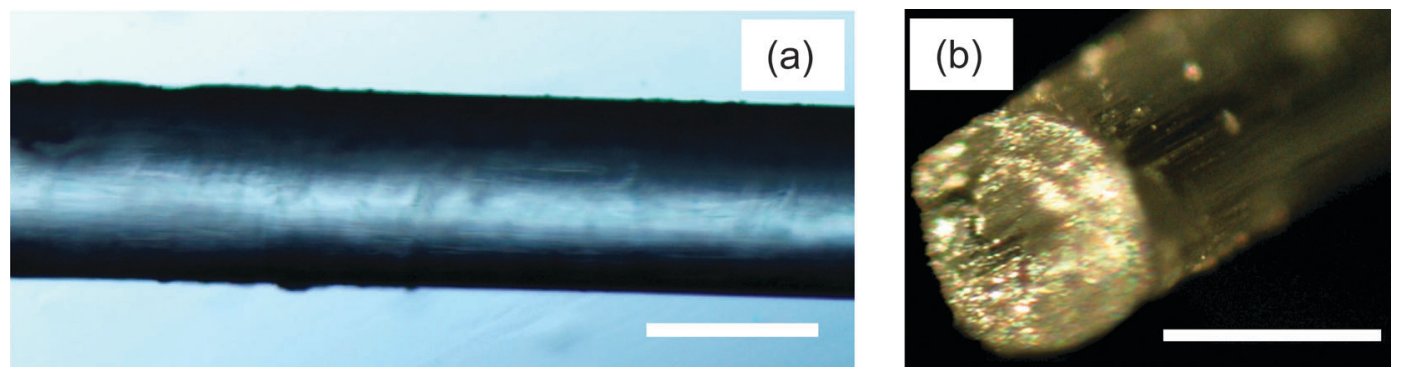

Fig. 1 (a) Lateral surface of a silkworm gut as observed with an optical microscope. (b) Cross sectional area of a silkworm silk. Calibration bars correspond to $500 \mu \mathrm{m}$ in both micrographs. Silkworm guts were prepared after incubation in a $2 \%$ acetic acid solution for 2 minutes. 

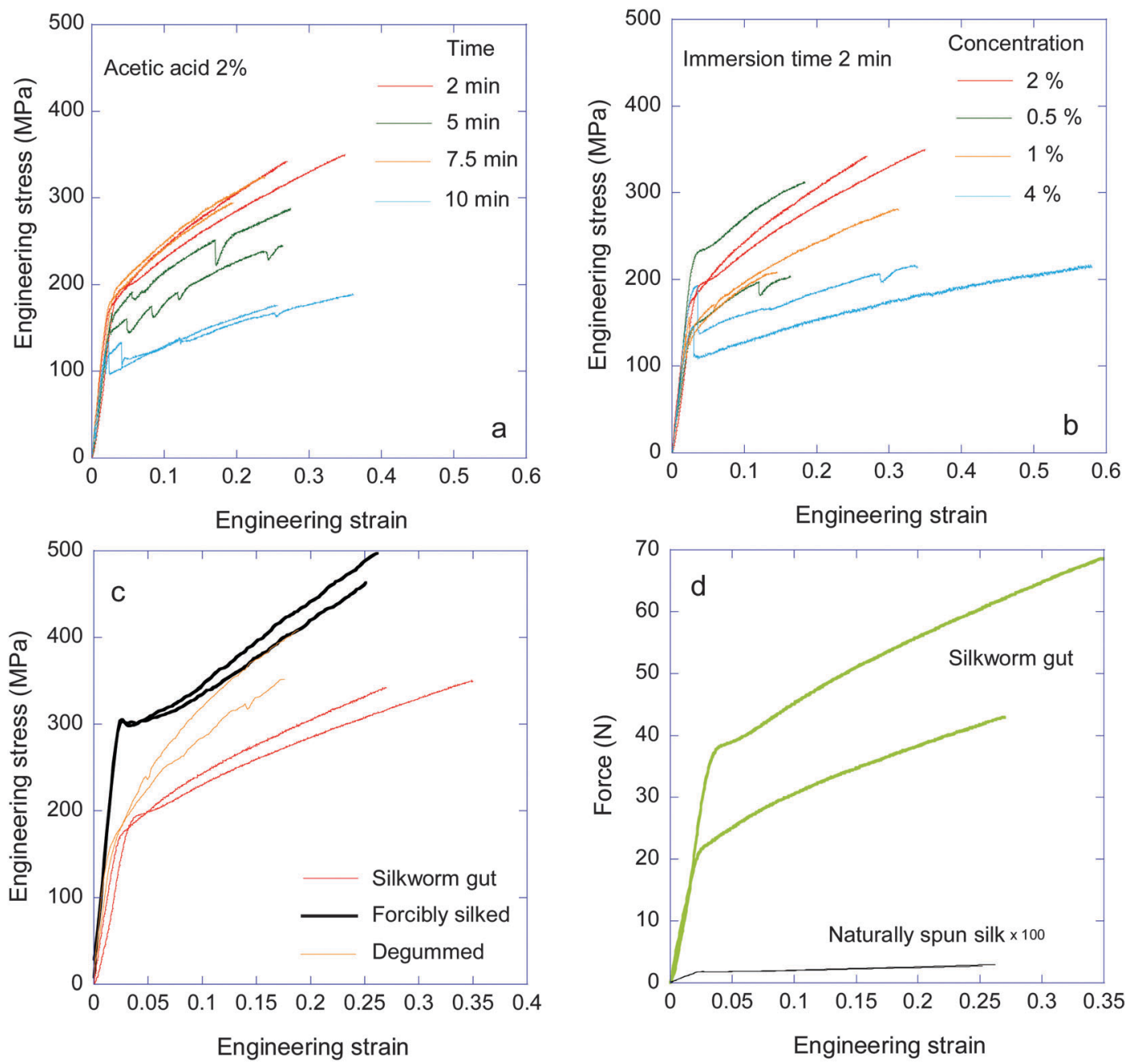

Fig. 2 Tensile properties of silkworm guts expressed as engineering stress-engineering strain curves. $2 \%$ acetic acid solution and immersion time of 2 minutes are taken as reference conditions. (a) Silkworm guts prepared in a $2 \%$ acetic acid solution for different times. (b) Silkworm guts prepared after 2 minutes of immersion in different acetic acid concentration solutions. (c) Comparison of the engineering stress-strain curves of representative silkworm guts and those of native silkworm silk fibers either as spun (forcibly silked, black lines) or after being subjected to degumming. (d) Comparison of the tensile properties of silkworm guts and naturally spun silkworm silk fibers in terms of force-engineering strain curves. Forces of the naturally spun fibers are multiplied by 100 in order to allow comparison in a single plot.

Table 1 Comparison of the mechanical parameters of forcibly silked (FS) and degummed $(\mathrm{Dg})$ silkworm silk and silk guts processed by immersion during 2 minutes in a $2 \%$ acetic acid solution. $E$ : elastic modulus; $\sigma_{\mathrm{y}}$ : yield stress; $\varepsilon_{\mathrm{u}}$ : strain at breaking; $\sigma_{\mathrm{u}}$ : tensile strength; and $W_{\mathrm{f}}$ : work to fracture. Values are presented as mean \pm standard error. The data corresponding to the FS and Dg silk were elaborated from the data of ref. 24 and 21, respectively. The data corresponding to the silkworm gut was obtained from four fibres produced after incubation in a 2\% acetic acid solution for 2 minutes

\begin{tabular}{lcllll}
\hline & $E(\mathrm{GPa})$ & $\begin{array}{l}\sigma_{\mathrm{y}} \\
(\mathrm{MPa})\end{array}$ & $\varepsilon_{\mathrm{u}}$ & $\begin{array}{l}\sigma_{\mathrm{u}} \\
(\mathrm{MPa})\end{array}$ & $\begin{array}{l}W_{\mathrm{f}} \\
\left(\mathrm{MJ} \mathrm{m}^{-3}\right)\end{array}$ \\
\hline FS silk & $13.9 \pm 0.1$ & $299 \pm 2$ & $0.25 \pm 0.01$ & $480 \pm 20$ & $92 \pm 4$ \\
Dg silk & $12 \pm 2$ & $140 \pm 8$ & $0.17 \pm 0.01$ & $380 \pm 30$ & $51 \pm 6$ \\
2\% 2 min & $7 \pm 1$ & $175 \pm 7$ & $0.34 \pm 0.03$ & $346 \pm 3$ & $80 \pm 10$ \\
silk gut & & & & &
\end{tabular}

the cross sectional area of both types of fibers is reflected in the corresponding force-strain curves, despite the force of the naturally spun fibers needing to be multiplied by 100 in order to become visible in the figure. A maximum force of $68.64 \mathrm{~N}$ (7.02 kgf) that corresponds to one of the curves presented in Fig. 1d was measured from silkworm silk guts.

\section{Microstructural characterization: X-ray diffraction}

In order to establish the parallelisms and differences between silkworm gut and native silk, the microstructures of both materials were assessed through X-ray diffraction. Probably, the most defining character of silkworm silk in microstructural terms is the presence of $\beta$-nanocrystallites, ${ }^{27-29}$ that result from the piling up of $\beta$-pleated sheets of regions with the motif -GAGAGS-. ${ }^{30}$ $\beta$-Nanocrystallites are extremely stable as determined by their stability at temperatures up to $250{ }^{\circ} \mathrm{C}^{16}$ and confer structural integrity to the fibers. ${ }^{31}$

Fig. 3 compares the XRD patterns of a degummed silkworm silk fiber ${ }^{16}$ (Fig. 3a) and that of a silkworm silk gut prepared after immersion in a $2 \%$ acetic acid bath for 2 minutes (Fig. $3 b$ ). 

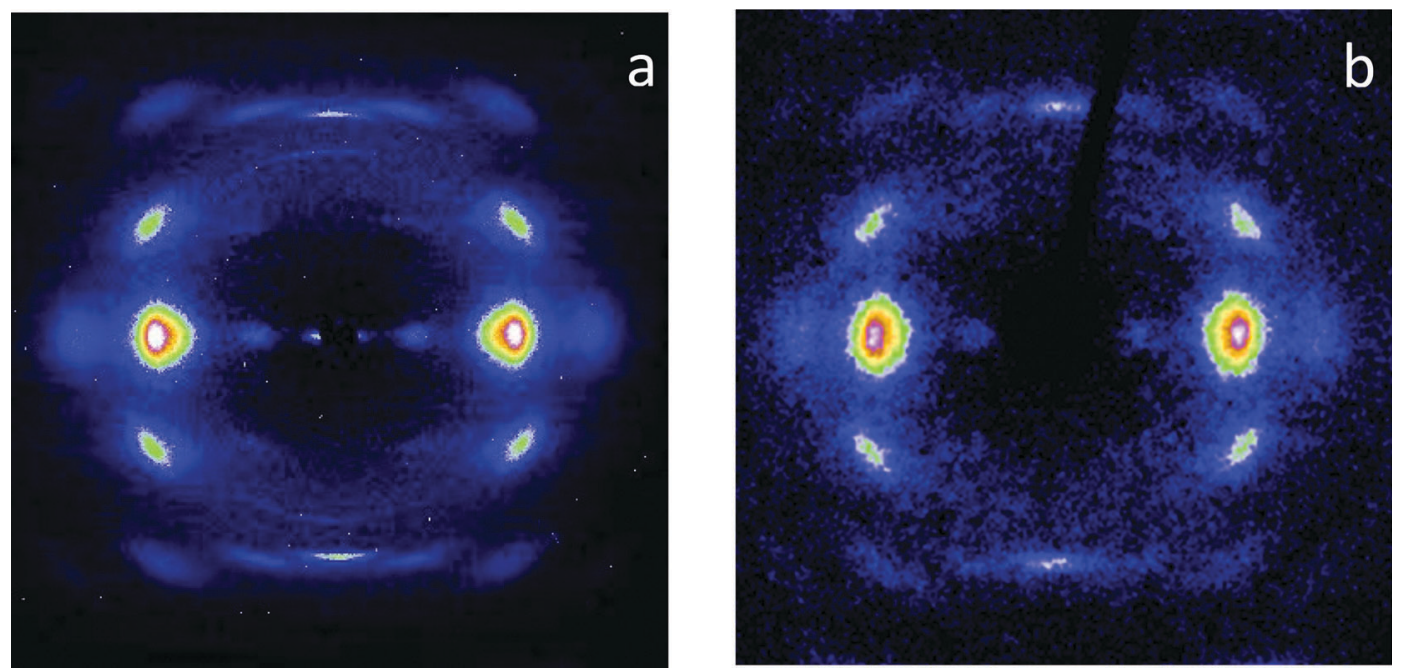

Fig. 3 XRD diffraction patterns of (a) degummed silkworm silk fiber, and (b) silkworm gut prepared by incubating the gland in a $2 \%$ acetic acid solution for 2 minutes.

Both patterns show similar diffraction spots that can be assigned to the (210), (020) and (002) crystallographic planes of an orthorhombic unit cell with parameters $a=0.95 \mathrm{~nm}$ (interchain hydrogen bonding - direction), $b=0.93 \mathrm{~nm}$ (piling up of $\beta$-sheets direction) and $c=0.70 \mathrm{~nm}$ (protein backbone direction). The identity of the unit cells indicates that fiber formation of silkworm silk guts is also the result of piling up $\beta$-pleated sheets that contain the -GAGAGS- motif. Consequently, this result proves that the only requirement for the formation of the nanocrystalline phase in silkworm silk is the combined action of an acid environment and tensile stress.

In contrast, significant differences are found between silkworm silk and silkworm gut when the rest of the parameters that define the crystalline phase (i.e. size of the nanocrystals, orientation of the nanocrystals and crystallinity) are compared (Table 2). In summary, silkworm silk gut is shown to be significantly less crystalline than silkworm silk, and the nanocrystals appear to be smaller and less oriented. In this regard, both parameters employed to measure crystallinity, $\chi$ and $X$, show that the crystalline fraction in silkworm silk gut represents

Table 2 Microstructural parameters of degummed (Dg) native silkworm silk and silkworm gut processed by submerging the sericigen gland for 2 minutes in a $2 \%$ acetic acid solution. $L[210], L[100]$ and $L[001]$ stand for the length of the nanocrystals along the corresponding crystallographic directions. FWHM: full width at half maximum of the (210) reflection. Crystallinity is measured alternatively as $\chi$ or as $X$, as defined in the Experimental section

\begin{tabular}{lcc}
\hline & Dg silk & $2 \% 2$ min silk gut \\
\hline$a(\mathrm{~nm})$ & $0.95 \pm 0.01$ & $0.94 \pm 0.02$ \\
$b(\mathrm{~nm})$ & $0.93 \pm 0.01$ & $0.93 \pm 0.03$ \\
$c(\mathrm{~nm})$ & $0.707 \pm 0.004$ & $0.70 \pm 0.01$ \\
$L[100](\mathrm{nm})$ & $11.3 \pm 0.4$ & $3.3 \pm 0.4$ \\
$L[010](\mathrm{nm})$ & $2.2 \pm 0.4$ & $2.5 \pm 0.2$ \\
$L[001](\mathrm{nm})$ & $15 \pm 1$ & $4.2 \pm 0.7$ \\
$(210)$ FWHM $\left(^{\circ}\right)$ & $12.1 \pm 0.1$ & $16.5 \pm 0.8$ \\
$\chi$ & $23 \pm 1 \%$ & $13 \pm 3 \%$ \\
$X$ & $45 \pm 1 \%$ & $22 \pm 3 \%$
\end{tabular}

approximately $50 \%$ of that found in silkworm silk. The orientation of the nanocrystals in silkworm silk gut as measured from the FWHM of the (210) reflection is smaller than that in naturally spun silkworm silk (i.e. the nanocrystals are less aligned with respect to the macroscopic axis of the fiber). The value of the FWHM observed in silkworm silk gut $\left(16.5^{\circ}\right)$ is comparable with the value observed in Argiope trifasciata MAS fibers, ${ }^{18}$ and significantly lower than the value of FWHM $=23^{\circ}$, measured from high performance regenerated silkworm silk fibers. ${ }^{32}$

Another significant difference between the crystalline phases of both types of fibers appears when comparing the sizes of the nanocrystals. The nanocrystals found in naturally spun silkworm silk are at least three times larger along the [100] and [001] directions. The [100] direction corresponds to the interchain direction of the $\beta$-pleated sheets (i.e. perpendicular to the protein backbone) and the [001] direction corresponds to the protein backbone. In contrast, the size of the nanocrystals along the [010] direction, which corresponds to the piling up direction, is comparable in both types of fibers. These differences allow inference that the $\beta$-pleated sheets that made up the $\beta$-nanocrystals in naturally spun silk are formed by the accretion of a larger number of protein chains which, in addition, are longer than those found in silkworm silk gut. The absence of such differences in the [010] direction indicates that the $\beta$-nanocrystals are formed as a consequence of the piling up of the same number of $\beta$-pleated sheets in both materials. Calculating the volume of the $\beta$-nanocrystals under the assumption of a parallelepiped geometry yields a value of $V \sim 370 \mathrm{~nm}^{3}$ for naturally spun silkworm silk, and $V \sim 35 \mathrm{~nm}^{3}$ for silkworm silk gut. Overall comparison of the microstructural features of naturally spun silkworm silk and silkworm silk gut indicates that the formation process of the latter is less efficient than the natural spinning process in terms of the creation of the nanocrystalline phase. The difference of over one order of magnitude in the size of the nanocrystals, but only of a factor 
of two in the crystalline fractions can be explained under the assumption that regions that lead to the formation of single nanocrystals in the natural material are fragmented into several smaller nanocrystals in silk guts.

\section{Microstructural characterization: infrared spectroscopy}

The analysis of the amorphous phase of silks is hampered by the absence of a technique which may provide the detailed information that X-ray diffraction provides on the crystalline phase. Vibrational spectroscopies, comprising infrared and Raman spectroscopies, are very often used with this objective, since these spectroscopies allow determination of the secondary structures of the silk proteins that constitute the fiber. ${ }^{33,34}$ In particular, the amide I peak, which corresponds essentially to the vibration of the $\mathrm{C}=\mathrm{O}$ group of the amide bond, is singularly sensitive to hydrogen bonding. Consequently, the deconvolution of this peak in its elementary contributions is customarily used to determine the secondary structure of the constituent protein chains. Preference of one vibrational spectroscopy over the other is usually a practical question influenced by the difficulty of obtaining the spectra for a given type of samples. Raman spectroscopy requires excitation of the vibrational modes of a sample with a laser which must be focused on the sample. Consequently, Raman spectroscopy is especially adequate for fibers with diameters in the range of a few microns. ${ }^{18}$ In the present study, the larger size of the sericigen glands and silkworm guts suggested the use of infrared spectroscopy, although this presents the disadvantage that water peaks present a certain degree of overlapping with the amide I peak (see below). This disadvantage, however, is compensated for by the possibility of monitoring silkworm gut formation from the gland to the final fiber, including several intermediate states.

Representative FTIR spectra corresponding to the amide I region of silkworm silk guts processed in acetic acid $2 \%$ solution for 2 minutes and obtained at different stages of the formation process are presented in Fig. $4 \mathrm{~b}-\mathrm{d}$. Fig. $4 \mathrm{~b}$ is labelled as a pre-gut fiber and was obtained by stretching the gland until it doubled its initial length (deformation, $e_{\mathrm{f}}=1$ ). This value is much smaller than the usual deformations reached when the formation of the silkworm silk gut is completed, since deformations of $e_{\mathrm{f}}=5-10$ are typically found. Fig. $4 \mathrm{c}$ corresponds to a pre-gut fiber allowed to dry in air for two hours before obtaining the FTIR spectrum and is labelled as a dry pre-gut fiber. Fig. 4d corresponds to a fully formed silkworm silk gut, observed immediately after formation. Finally, Fig. 4 also includes an FTIR spectrum of a $2 \%$ aqueous solution of acetic acid in water (Fig. 4a) and of a natural silkworm silk fiber after being subjected to a degumming process (Fig. 4e). All spectra were normalized and the Gaussian functions that correspond to the elementary contributions are shown in each plot.

Fig. 4a shows that an acetic acid solution presents a significant contribution at values close to the amide I peak. In particular, two elementary contributions at approximately 1640 and $1680 \mathrm{~cm}^{-1}$ are found. Identical spectra were obtained from distilled water and from the native gland, even after being immersed in $2 \%$ acetic acid for 2 minutes. This spectrum was taken as a reference, since it was found that all spectra showed two contributions at approximately these wavelengths that were consistently assigned to the presence of water molecules in the fiber. The absence of significant differences between this spectrum and that obtained from the gland indicates that the technique does not have high enough resolution so as to discriminate between the contribution of the soluble proteins in the gland and that of the water molecules.

The spectrum of the pre-gut fiber (Fig. 4b) shows the two contributions found in Fig. 4a and an additional contribution at approximately $1614 \mathrm{~cm}^{-1}$. This contribution was previously assigned to the $\beta$-pleated sheets ${ }^{33}$ and indicates that the formation of this secondary structure is one of the earliest events during the formation process of the fiber, probably related to the formation of $\beta$-nanocrystals. Two more contributions appear at approx. 1660 and $1698 \mathrm{~cm}^{-1}$ after the pre-gut fiber is allowed to dry for two hours (Fig. 4c). These contributions can be assigned to $3_{1}$ helices and $\beta$-pleated sheets, respectively. ${ }^{35,36}$ The absence of these contributions in the pre-gut fiber before drying might be attributed to either the lower resolution that results from the presence of the large water peaks or by the organization of the protein chains in these secondary structures after water removal. Unfortunately, the characteristic vibration of the random coil structure is found in the range $1638-1655 \mathrm{~cm}^{-1}$ which fully overlaps with one of the contributions of water.

Fig. $4 \mathrm{~d}$ presents the spectrum of a fully formed silkworm silk gut, in which the contributions previously found in the dried pre-gut fiber can also be identified: two contributions assigned to water (1640 and $1680 \mathrm{~cm}^{-1}$ ), $3_{1}$ helices $\left(1660 \mathrm{~cm}^{-1}\right.$ ), and $\beta$-pleated sheets $\left(1614 \mathrm{~cm}^{-1}\right.$ and $\left.1698 \mathrm{~cm}^{-1}\right)$. In addition, two further contributions are also found at $1598 \mathrm{~cm}^{-1}$ and $1630 \mathrm{~cm}^{-1}$. These contributions can be assigned to $\beta$-sheets and to loosely packed $\beta$-sheets (sometimes called intramolecular $\beta$-sheets), respectively. It is also apparent that $\beta$-pleated sheets represent the main contribution to the amide I peak of the silk gut, since the addition of the three peaks assigned to $\beta$-sheets represents approx. $40 \%$ of the total area.

Finally, Fig. 4e shows the spectrum of a degummed naturally spun silkworm silk fiber. The main contributions previously found in Fig. 4d are also found here, although some significant differences are observed when comparing the spectra. In this regard, native silk does not show a contribution at $1630 \mathrm{~cm}^{-1}$ corresponding to intramolecular $\beta$-sheets. Instead, a new contribution is observed at approx. $1690 \mathrm{~cm}^{-1}$ which can be assigned to $\beta$-turn structures. ${ }^{37,38}$ Addition of the three contributions assigned to $\beta$-pleated sheets represents approx. $55 \%$ or the total area, which is a value very close to the usually accepted value of $60 \%$ for the fraction of proteins forming $\beta$-nanocrystals in silkworm silk fibers. ${ }^{39}$

FTIR analysis of silkworm silk gut at different stages during the formation process allows the establishment of some of the basic facts about the transition between the protein solution in the gland and the solid fiber. Formation of $\beta$-sheets, probably piling up into $\beta$-nanocrystals, appears to be the critical event that prompts the formation of the solid fiber. Unfortunately, 

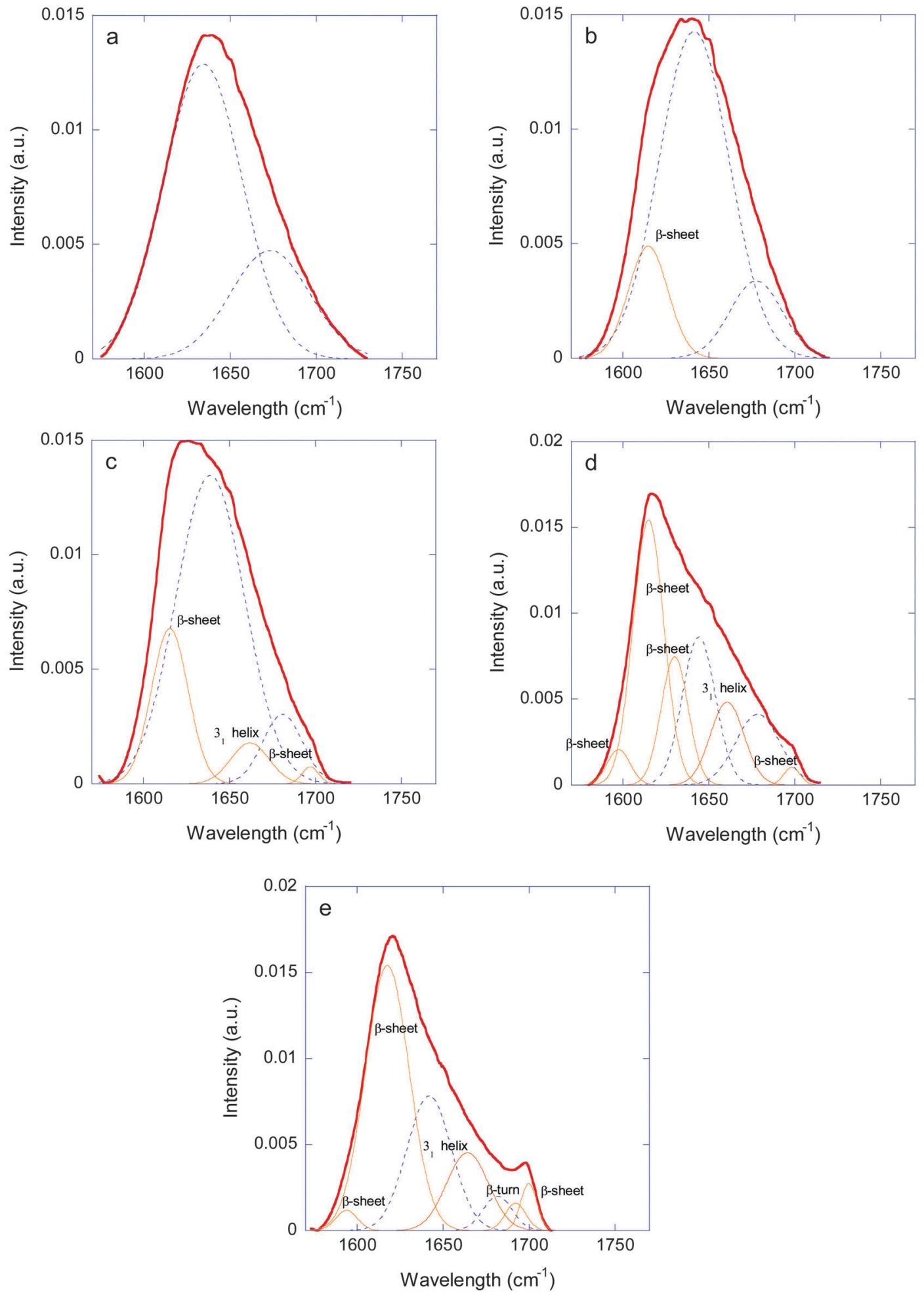

Fig. 4 FTIR spectra of the amide I region of silkworm guts. (a) Aqueous solution of $2 \%$ acetic acid in water. Identical spectra were obtained from distilled water and from the gland after being immersed in a $2 \%$ acetic acid solution. (b) Silkworm pre-gut ( $2 \%$ acetic acid, 2 min) obtained by stretching the gland to a formation strain of $e_{f}=1$. The spectrum was recorded immediately after forming the pre-gut fiber. (c) Silk pre-gut ( $2 \%$ acetic acid, 2 min) dried for 2 hours in air before obtaining the spectrum. (d) Silkworm gut prepared by incubating the gland in 2\% acetic acid solution for 2 minutes. (e) FTIR spectrum of a degummed naturally spun silk fiber. All spectra are normalized to unit area. The elementary contributions are modelled by Gaussians. The two contributions found in the spectrum of the acetic acid solution are marked with blue and discontinuous lines in all spectra.

the presence of an important contribution due to water molecules in the region of the amide I peak does not allow gain of any conclusive information about the other secondary structures that are formed by the fibroin proteins at this stage. 
In this regard, the presence of a significant contribution assigned to $3_{1}$ helices in the dried pre-gut fiber might indicate that this secondary structure might play an important role in the amorphous regions of the fiber. The secondary structure of silkworm silk gut after being completely formed is dominated by the presence of $\beta$-pleated sheets. In addition, a minor contribution that can be assigned to $3_{1}$ helices is also found. However, a significant proportion of the $\beta$-pleated sheets seems to correspond to intramolecular $\beta$-sheets, i.e. regions where the packing of the protein chains is not as tight in the proper $\beta$-nanocrystals. In contrast, this contribution is not identified in the degummed fiber, whose spectrum is dominated by $\beta$-pleated sheets with minor contributions of $3_{1}$ helices and $\beta$-turns. The results obtained by FTIR support the original hypothesis presented from analysis of the XRD data and suggest that the aggregation process that leads to the appearance of $\beta$-nanocrystals is somewhat inhibited during the formation process of silkworm silk gut. A less efficient aggregation process would lead to regions with $\beta$-pleated secondary structure, which are not incorporated into the $\beta$-nanocrystals.

\section{Forces involved in silkworm silk gut formation}

The experimental setup for forming silkworm silk guts from silk glands allows monitoring of the forces and displacements associated with the process and, consequently, provides information on details inaccessible in the natural spinning system. Fig. 5 shows representative curves of the forces measured during silkworm silk gut formation $v$ s. formation engineering strain ( $F-e_{\mathrm{f}}$ curves). Formation strain, $e_{\mathrm{f}}$, is defined as the ratio between the increase in length of the gland and its initial length fixed to $60 \mathrm{~mm}$. It is observed that the glands reach values of strain between 5 and 10, which implies a final length of the fiber after formation of over $600 \mathrm{~mm}$. Standard formation conditions were taken again as using $2 \%$ acetic acid solution for 2 minutes of immersion time. Fig. 5a shows the $F-e_{\mathrm{f}}$ plots measured during the formation process of fibers treated in a $2 \%$ acetic acid solution,
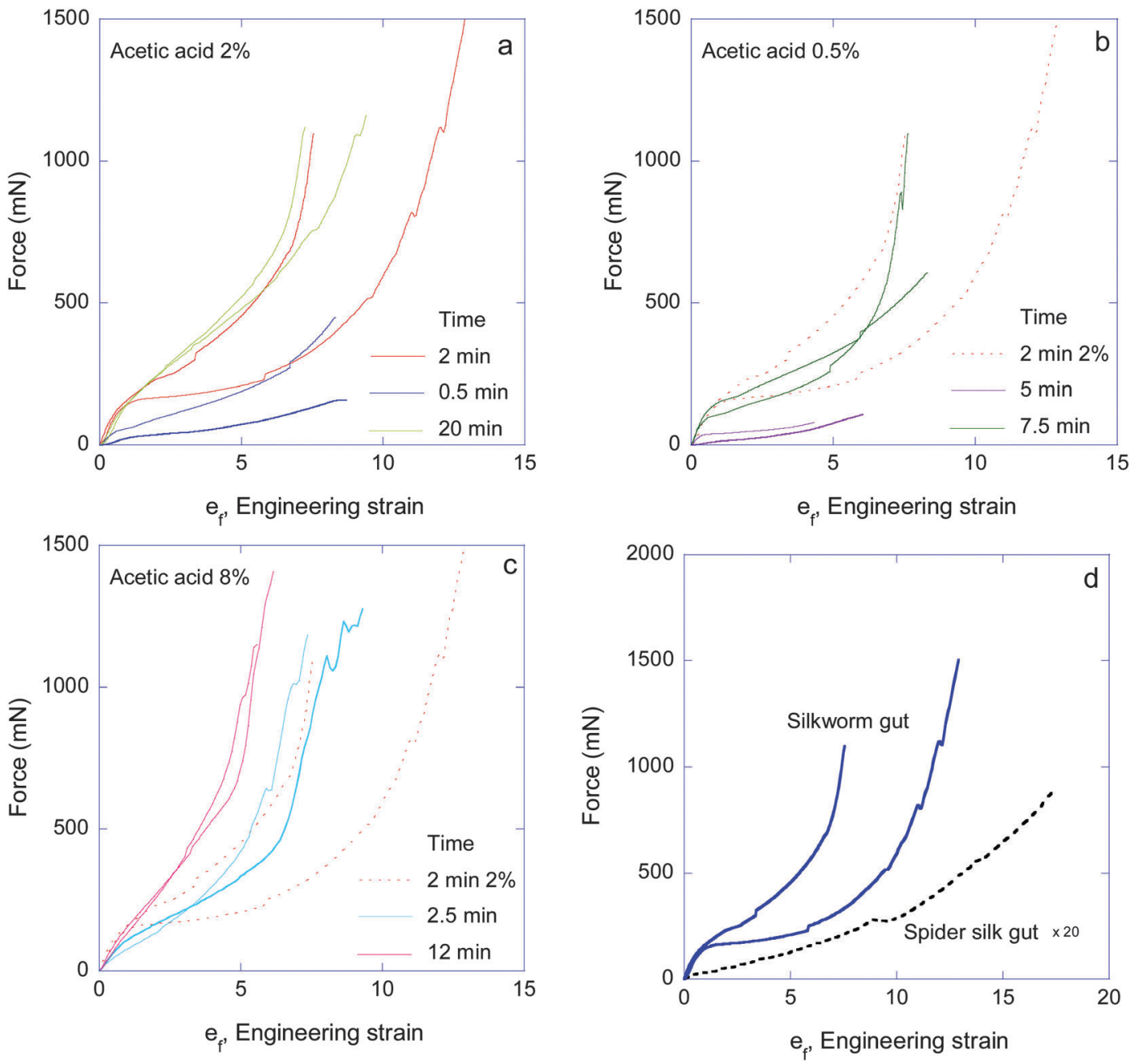

Fig. 5 Mechanical behavior of the gland exhibited during the silkworm gut formation process. $2 \%$ acetic acid solution and immersion time of 2 minutes are taken as reference conditions. (a) Formation of silkworm gut in $2 \%$ acetic acid solution and different times. (b) Formation of silkworm gut in $0.5 \%$ acetic acid solution and different times. (c) Formation of silkworm gut in $8 \%$ acetic acid solution and different times. (d) Comparison of the force-strain curves measured during the formation process of silkworm gut (continuous line) and spider silk gut (broken line). The force of the spider silk gut is multiplied by 20 to allow representation in the same plot. 
Fig. 5b those formed in $0.5 \%$ acetic acid solution and Fig. $5 \mathrm{c}$ those formed in $8 \%$ acetic acid solution. A certain tendency towards stiffer curves (i.e. larger values of force for a given value of strain) is observed at higher concentrations of acetic acid and/or longer times. In contrast, forces are significantly reduced in treatments that use lower acetic acid concentrations and/or shorter times.

Although a detailed assessment of the stresses involved in the formation process is far from straightforward, due to problems related with the measurement of the cross sectional area, ${ }^{15}$ an acceptable estimation of the upper limit can be obtained if the cross sectional area of a fully formed fiber is used. In this regard, it was found that a solid fiber was formed when the gland doubled its initial length (i.e. strain, $e_{\mathrm{f}}=1$ ). If the forces measured at this value of strain are combined with the cross sectional area that corresponds to the diameters of the fibers ( $D=500-600 \mu \mathrm{m})$, the formation stresses are estimated to lie in the range of $s_{\mathrm{f}}=200-1000 \mathrm{kPa}$. These values compare well with the formation stress measured from spider silk gut, $s_{\mathrm{f}}=700 \mathrm{kPa}$, and are significantly smaller than previous estimations of the stresses exerted on the dope during the fiber formation process, $s_{\mathrm{f}}=20-40 \mathrm{MPa} .{ }^{40-42}$ These latter values had been obtained by either analysing the rheological behaviour of the protein solution in the gland ${ }^{40,41}$ or by direct measurement of the forces involved in the forced silking process. ${ }^{42}$ Discrepancy between the values obtained from the analysis of the silk guts and those obtained from the natural spinning process might indicate that forces are required in the latter for other functions distinct to inducing the solidification of the fiber.

Fig. 5d compares representative force $v s$. strain curves obtained during the formation processes of silkworm and spider silk guts (forces of the spider silk gut are multiplied $\times 20$ in order to allow the representation of all the data in a single plot). It is apparent that spider silk gut reaches higher values of maximum strain during formation as illustrated in Fig. $5 \mathrm{~d}$ and further supported by the comparison of Fig. 5 and the corresponding results for spider silk gut prepared under different conditions. ${ }^{15}$ Besides the difference in maximum strain there is another significant difference between both silk guts that is apparent at low values of strain. The $F-e_{\mathrm{f}}$ curves of spider silk gut correspond to an elastomeric material ${ }^{43}$ and, consequently, it is assumed that their properties are controlled by forces of an entropic origin. In contrast, the behaviour of silkworm silk gut at low values of formation strain does not correspond to a typical elastomeric material due to the high stiffness shown by the $F-e_{\mathrm{f}}$ curve at strains $e_{\mathrm{f}} \leq 1$. More experiments will be required to identify the origin of these distinct behaviours in spider and silkworm silk guts. However, a very tentative hypothesis can be proposed by taking into account that entropic behaviour is related to the unfolding of chains, while enthalpic (i.e. non-elastomeric) is related to the stretching of bonds. In this regard, the initial enthalpic behaviour of silkworm silk gut might be the consequence of the deformation of the incipient $\beta$-nanocrystals that form during initial stretching as found by FTIR (Fig. 4b), before the rest of the fibroin proteins begin to unfold.
The possibility of obtaining fibers directly from the gland through the silkworm silk gut formation process confirms the basic assumptions of the accepted model proposed to explain the spinning of silk fibers. In this regard it is found that the fibers are formed exclusively if the whole gland is initially exposed to an acidic environment. Neutral or basic solutions do not lead to the formation of the fiber, even if the gland is subjected to mechanical stress. The formation of the fiber requires, in addition, that the acidified gland be exposed to mechanical stress. The interplay of an acidic environment and the appearance of mechanical stress on the dope had been previously identified as critical conditions in the natural spinning system, ${ }^{44,45}$ and might represent a general mechanism in the formation process of natural fibers. ${ }^{46}$ The model assumes that the fibroin proteins show either random coil or $\alpha$-helix conformations when found in solution in the gland. Proton pumps found in the distal part of the duct are supposed to increase the $\mathrm{pH}$ of the dope, ${ }^{47}$ and the variation of $\mathrm{pH}$ unleashes a self-assembly process of fibroins controlled by the presence of $\mathrm{pH}$-switches in the $\mathrm{N}$ - and C-terminal domains of the proteins. ${ }^{48,49}$ The action of mechanical stress on these self-assembled structures induces a conformational change to the $\beta$-pleated secondary structure and the formation of $\beta$-nanocrystals. $\beta$-Nanocrystals confer structural integrity to the fibers and allow insoluble solid fibers from an aqueous solution to be obtained. This model is basically confirmed from the mechanical and microstructural data presented above. However, in contrast to the formation process of spider silk gut that yields fibers with the same properties as those exhibited by the naturally spun material, silkworm silk guts present some differences when compared with the native material. These differences are supposed to be related to the smaller size of the $\beta$-nanocrystals and reduced crystallinity of silkworm gut compared with the values exhibited by naturally spun silkworm silk. Consequently, the native spinning system of silk must involve some additional mechanisms that guarantee the correct formation of the crystalline phase during processing.

\section{Conclusions}

Silkworm silk gut processing represents an alternative to native spinning of silk for the production of high performance fibers. Silkworm gut fibers within a relatively wide range of tensile properties are obtained by immersion in an acidic solution and stretching. The tensile properties are influenced by the formation conditions although there seems to be a range of acetic acid concentrations and immersion times that lead to fibers with comparable properties. Silk guts are more compliant and do not reach such high values of tensile strength, but can reach higher values of strain at breaking when compared with the natural material. The values of tensile strength and strain at breaking found in silk guts yield values of work to fracture comparable to native silkworm silk. The much larger cross sectional area of silk guts implies that the forces that these fibers can sustain are four orders of magnitude larger than those sustained by native silkworm silk fibers. 
In addition, the availability of the silk gut production route allows gain of a deeper insight in the factors that influence the spinning process. In this regard, measuring the stress involved in the formation process indicates that the fiber is formed at a stress well below a value of $1000 \mathrm{kPa}$. Besides, it was found that the formation of $\beta$-pleated sheets is the initial conformational change undergone by the protein chains during the formation process. $\beta$-Nanocrystals in both the silk gut and native silk have the same unit cells, although the size of the nanocrystals in silk gut is much smaller than that found in the native material. Microstructural data suggest that differences might be related to the formation of smaller nanocrystals in silk gut. The presence of larger nanocrystals in the native silk suggests that the natural spinning route involves some additional mechanisms that improve the quality of the crystalline phase.

\section{Authors contributions}

JLC, SDAC and AALP bred the silkworms and prepared the material. RM and GRP performed the mechanical characterization. RM and NMB performed the X-ray diffraction and infrared analysis of the data, respectively. JLC and JPR proposed the experiments, coordinated the research and wrote the main text. All authors contributed to the data analysis and reviewed the manuscript.

\section{Acknowledgements}

The authors are grateful to José Miguel Martínez for his help with the artwork. The work was funded by the Ministerio de Economía y Competitividad (Spain) through project MAT201238412-C02-01, by the Fundación Marcelino Botín and by Banco Santander through its Santander Universities Global Division. Dr A. Abel Lozano-Pérez's research contract was partially supported ( $80 \%)$ by the FEDER Operative Program of the Region of Murcia 2007-2013.

\section{References}

M. Heim, D. Keerl and T. Scheibel, Angew. Chem., Int. Ed., 2009, 48, 3584-3596.

H. J. Jin and D. L. Kaplan, Nature, 2003, 424, 1057-1061, DOI: $10.1038 /$ nature01809.

F. Vollrath and D. P. Knight, Nature, 2001, 410, 541-548.

C. Y. Hayashi, N. H. Shipley and R. V. Lewis, Int. J. Biol. Macromol., 1999, 24, 271-275.

5 G. Gronau, S. T. Krishnaji, M. E. Kinahan, T. Giesa, J. Y. Wong, D. L. Kaplan and M. J. Buehler, Biomaterials, 2012, 33, 8240-8255, DOI: 10.1016/j.biomaterials.2012.06.054.

Z. Z. Shao, F. Vollrath, Y. Yang and H. C. Thogersen, Macromolecules, 2003, 36, 1157-1161, DOI: 10.1021/ma0214660.

T. Scheibel, Microb. Cell Fact., 2004, 3, 14, DOI: 10.1186/ 1475-2859-3-14.

8 L . M a r d e n , National Geographic Magazine, 1951, 100, 100-108.

A. M. C. Humphries, Postgrad. Med. J., 1949, 483-488.
L. H. H. Martin, The American Fly Fisher, 1991, 17, 3-7.

P. Schullery, The American Fly Fisher, 2006, 32, 2-9.

12 G. A l t m a n, R. H o r a n, H. L u, J . M o r e a u, I. M a r

$\mathrm{t}$ i n, J . R i c h m o n d

and D. Kaplan, Biomaterials, 2002, 23, 4131-4141, DOI: 10.1016/S0142-9612(02)00156-4.

C. Vepari and D. Kaplan, Prog. Polym. Sci., 2007, 32, 991.

Y. Wang, H. Kim, G. Vunjak-Novakovic and D. Kaplan, Biomaterials, 2006, 27, 6064.

P. Jiang, N. Mari-Buye, R. Madurga, M. Arroyo-Hernandez, C. Solanas, A. Ganan, R. Daza, G. R. Plaza, G. V. Guinea, M. Elices, J. L. Cenis and J. Perez-Rigueiro, Sci. Rep., 2014, 4, 7326, DOI: 10.1038/srep07326.

A. Martel, M. Burghammer, R. J. Davies and C. Riekel, Biomacromolecules, 2007, 8, 3548-3556, DOI: 10.1021/bm700935w.

C. Riekel, C. Branden, C. Craig, C. Ferrero, F. Heidelbach and M. Muller, Int. J. Biol. Macromol., 1999, 24, 179-186.

18 G. R. Plaza, J. Pérez-Rigueiro, C. Riekel, G. B. Perea, F. AgullóRueda, M. Burghammer, G. V. Guinea and M. Elices, Soft Matter, 2012, 8, 6015-6026, DOI: 10.1039/C2SM25446H.

H. P. Klug and L. Alexander, X-ray Diffraction Procedures for Polycrystalline and Amorphous Materials, John Wiley \& Sons, New York, USA, 2nd edn, 1974.

A. Misra, R. S. Stein, C. Chu, G. L. Wilkes and A. B. Desai, J. Polym. Sci., Part C: Polym. Lett., 1975, 13, 303-312.

J. Perez-Rigueiro, C. Viney, J. Llorca and M. Elices, J. Appl. Polym. Sci., 1998, 70, 2439-2447.

K. K. Chawla, Fibrous Materials, Cambridge University Press, Cambridge, U.K., 1998.

J. G. Hardy, L. M. Romer and T. R. Scheibel, Polymer, 2008, 49, 4309-4327, DOI: 10.1016/j.polymer.2008.08.006.

J. Perez-Rigueiro, M. Elices, J. Llorca and C. Viney, J. Appl. Polym. Sci., 2001, 82, 1928-1935.

Z. Z. Shao and F. Vollrath, Nature, 2002, 418, 741, DOI: 10.1038/418741a.

J. Perez-Rigueiro, M. Elices, J. Llorca and C. Viney, J. Appl. Polym. Sci., 2002, 84, 1431-1437, DOI: 10.1002/app.10366.

R. E. Marsh, R. B. Corey and L. Pauling, Biochim. Biophys. Acta, 1955, 16, 1-34.

Y. Takahashi, Silk Polymers, 1994, 544, 168-175.

Y. Takahashi, M. Gehoh and K. Yuzuriha, Int. J. Biol. Macromol., 1999, 24, 127-138.

Q. Y. Xia, Z. Y. Zhou, C. Lu, D. J. Cheng, F. Y. Dai, B. Li, P. Zhao, X. F. Zha, T. C. Cheng, C. L. Chai, G. Q. Pan, J. S. Xu, C. Liu, Y. Lin, J. F. Qian, Y. Hou, Z. L. Wu, G. R. Li, M. H. Pan, C. F. Li, Y. H. Shen, X. Q. Lan, L. W. Yuan, T. Li, H. F. Xu, G. W. Yang, Y. J. Wan, Y. Zhu, M. D. Yu, W. D. Shen, D. Y. Wu, Z. H. Xiang, J. Yu, J. Wang, R. Q. Li, J. P. Shi, H. Li, G. Y. Li, J. N. Su, X. L. Wang, G. Q. Li, Z. J. Zhang, Q. F. Wu, J. Li, Q. P. Zhang, N. Wei, J. Z. Xu, H. B. Sun, L. Dong, D. Y. Liu, S. L. Zhao, X. L. Zhao, Q. S. Meng, F. D. Lan, X. G. Huang, Y. Z. Li, L. Fang, C. F. Li, D. W. Li, Y. Q. Sun, Z. P. Zhang, Z. Yang, Y. Q. Huang, Y. Xi, Q. H. Qi, D. D. He, H. Y. Huang, X. W. Zhang, Z. Q. Wang, W. J. Li, Y. Z. Cao, Y. P. Yu, H. Yu, J. H. Li, J. H. Ye, H. Chen, Y. Zhou, B. Liu, J. Wang, J. Ye, H. Ji, S. T. Li, P. X. Ni, J. G. Zhang, Y. Zhang, H. K. Zheng, B. Y. Mao, W. Wang, 
C. Ye, S. G. Li, J. Wang, G. K. S. Wong and H. M. Yang, Science, 2004, 306, 1937-1940, DOI: 10.1126/science.1102210.

S. Keten, Z. P. Xu, B. Ihle and M. J. Buehler, Nat. Mater., 2010, 9, 359-367, DOI: 10.1038/NMAT2704.

G. R. Plaza, P. Corsini, E. Marsano, J. Perez-Rigueiro, L. Biancotto, M. Elices, C. Riekel, F. Agullo-Rueda, E. Gallardo, J. M. Calleja and G. V. Guinea, Macromolecules, 2009, 42, 8977-8982, DOI: 10.1021/ma9017235.

C. Pellerin, M. E. Rousseau, M. Cote and M. Pezolet, Macromol. Symp., 2005, 220, 85-98, DOI: 10.1002/masy.200550207.

F. Paquet-Mercier, T. Lefevre, M. Auger and M. Pezolet, Soft Matter, 2013, 9, 208-215, DOI: 10.1039/c2sm26657a.

H. Teramoto and M. Miyazawa, Biomacromolecules, 2005, 6, 2049-2057, DOI: 10.1021/bm0500547.

X. Chen, D. P. Knight, Z. Z. Shao and F. Vollrath, Polymer, 2001, 42, 9969-9974.

A. Dong, P. Huang and W. S. Caughey, Biochemistry, 1990, 29, 3303-3308, DOI: 10.1021/bi00465a022.

D. Wilson, R. Valluzzi and D. Kaplan, Biophys. J., 2000, 78, 2690-2701.

D. B. Gillespie, C. Viney and P. Yager, Silk Polymers: Materials
C. Holland, A. E. Terry, D. Porter and F. Vollrath, Nat. Mater., 2006, 5, 870-874, DOI: 10.1038/nmat1762.

N. Kojic, J. Bico, C. Clasen and G. H. McKinley, J. Exp. Biol., 2006, 209, 4355-4362, DOI: 10.1242/jeb.02516.

M. Elices, G. V. Guinea, G. R. Plaza, J. I. Real and J. PerezRigueiro, J. Mater. Res., 2006, 21, 1931-1938, DOI: 10.1557/ JMR.2006.0240.

J. M. Gosline, M. W. Denny and M. E. Demont, Nature, 1984, 309, 551-552.

E. Iizuka, Appl. Polym. Symp., 1985, 173-185.

A. Rising and J. Johansson, Nat. Chem. Biol., 2015, 11, 309-315, DOI: 10.1038/NCHEMBIO.1789.

N. R. Martinez Rodriguez, S. Das, Y. Kaufman, J. N. Israelachvili and J. H. Waite, Biofouling, 2015, 31, 221-227, DOI: 10.1080/ 08927014.2015.1026337.

F. Vollrath, D. P. Knight and X. W. Hu, Proc. R. Soc. London, Ser. B, 1998, 265, 817-820.

G. Askarieh, M. Hedhammar, K. Nordling, A. Saenz, C. Casals, A. Rising, J. Johansson and S. D. Knight, Nature, 2010, 465, 236-239.

F. Hagn, L. Eisoldt, J. G. Hardy, C. Vendrely, M. Coles, T. Scheibel and H. Kessler, Nature, 2010, 465, 239-242. 